\title{
Genomics of Renal Cell Cancer - Does It Provide Breakthrough?
}

\author{
László KOPPER, ${ }^{1}$ József TÍMÁR ${ }^{2}$ \\ ${ }^{1} 1$ st Department of Pathology and Experimental Cancer Research, Semmelweis University, \\ ${ }^{2}$ Department of Tumor Progression, National Institute of Oncology, Budapest, Hungary
}

It is a strong hope that the more we characterize the pathways in an individual tumor, the better we will be able to evaluate the response to a specific therapy. Different array technologies could be powerful tools to achieve this goal, i.e. selecting patients on the basis of the genomic and/or proteomic profiles who would really benefit from the target-designed therapy. Genomic analysis of RCC accumulated ample of data which now can be exploited in clinical management of a previously almost uncontrollable disease. Beside the previously identified genetic abnormalities (VHL, MET, EGFR), CAIX seems to be a novel molecular marker of RCC. Array studies also outlined a small set of tumor markers, vimentin, galectin-3, CD74 and parvalbumin, which can define the individual histologic subtypes of RCC. We are at the beginning to take advantage of the genomic results. Some new approaches will interfere with the progression of RCC (anti-VEGF, anti-VEGFR or antiEGFR therapies). Further novel molecular targets are available, such as HIF, HSP90 or the IFN-regulated genes, which can be used to the fine-tuning of RCC therapy. (Pathology Oncology Research Vol 12, No 1, 5-11)

Key words: renal cell cancer, genomics, prognostic markers, targeted therapy

\section{Introduction}

Renal cell cancer (RCC) accounts for $\sim 3 \%$ of human malignancies and its incidence appears to be rising. The prognosis of RCC is mainly related to stage (5-year survival is $>90 \%$ in stage I, but $<20-30 \%$ in stage IV). Unfortunately many RCC masses remain asymptomatic and nonpalpable until they are advanced. RCC is not a single disease, it has several morphological subtypes. Clear cell RCC accounts for $\sim 80 \%$ of cases, followed by papillary RCC (10-15\%), chromophobe RCC (5\%), collecting duct RCC $(<1 \%)$ and unclassified $(<2 \%)$. Sarcomatoid features can appear in all forms as a sign of active epithelial-mesenchymal transition. It is not viewed as a separate histological entity, but as a highly aggressive form of tumor dedifferentiation with an extremely poor prognosis. ${ }^{21}$ Most cases of $\mathrm{RCC}$ are sporadic, with main risk factors as smoking, obesity, occupational exposures, but hereditary RCC syndromes $(<3 \%)$ have also major clinical implications.

Received: Jan 10, 2006; accepted: Febr 20, 2006

Correspondence: László KOPPER, MD, PhD, 1st Department of Pathology and Experimental Cancer Research, Semmelweis University, Üllói út 26., Budapest, H-1085, Hungary; Tel/fax: +36 1 3170891, E-mail: kopper@korb1.sote.hu
RCC was a therapy resistant cancer, especially in its advanced stage where, beside surgical intervention, moderate improvements were achieved by other modalities. Recently, molecular analysis of RCC revealed characteristic genetic alterations connected to specific histologic subtypes. According to expectations these studies will lead to the identification of new targets for a more effective therapy. This trend has just started. ${ }^{46,54}$ This review summarizes the state-of-art of RCC genomics and demonstrates how this knowledge could be translated to a more efficient clinical management of this disease.

\section{Studies on single gene or protein changes}

Susceptibility genes (inheritance, family accumulation)

Identification of genes responsible for inherited RCC improved the understanding of renal tumorigenesis including sporadic RCC, and raised new therapeutic approaches. To date, ten familial syndromes have been associated with one or more of the various histological subtypes of RCC. They follow an autosomal dominant trait (Table 1). ${ }^{26,37,39}$ Almost all of these syndromes have numerous clinical manifestations besides RCC. It is critical to suspect an inherited syndrome when RCC is bilater- 
Table 1. Familial syndromes associated with renal cell carcinoma

\begin{tabular}{|c|c|c|}
\hline Renal tumor & Disease & Gene \\
\hline \multicolumn{3}{|l|}{ Clear cell RCC } \\
\hline bilateral, multiple & von Hippel-Lindau* & VHL (3p25-26) \\
\hline unilateral, solitary & familial clear cell RCC & $\begin{array}{l}\text { unknown, ? vHL } \\
\text { unknown }\end{array}$ \\
\hline Clear cell RCC & hereditary paraganglioma & SDHB (1p36) \\
\hline Clear cell RCC, angiomyolipomas & tuberous sclerosis & $\begin{array}{l}\text { TSC1 (9q34), } \\
\text { TSC2 (16p13) }\end{array}$ \\
\hline \multicolumn{3}{|l|}{ Papillary RCC } \\
\hline type 1 , bilateral, multiple & hereditary papillary $R C C^{*}$ & MET (7q31) \\
\hline type 2 , unilateral, solitary & hereditary leiomyomatosis RCC* & FH (1q42-43) \\
\hline Papillary RCC, renal hamartomas, Wilm's tumor & hyperparathyroidism-jaw tumor & HRPT (1q25-32) \\
\hline Papillary RCC, oncocytoma & familial papillary thyroid cancer & $?(1 \mathrm{q} 21)$ \\
\hline $\begin{array}{l}\text { Various RCC } \\
\text { oncocytic-chromophobe RCC } \\
\text { chromophobe, clear cell RCC } \\
\text { oncocytomas }\end{array}$ & Birt-Hogg-Dubé* & BHD (17p11.2) \\
\hline
\end{tabular}

*the most common forms

al and/or multiple, or appears at a younger age. The diagnosis can be confirmed by the analysis of the main predisposing genes (VHL, MET, FH, BHD).

Von Hippel-Lindau (VHL) disease is resulted by the germline mutations of VHL tumor suppressor gene. pVHL takes part in the proteasomal degradation of hypoxiainducible factor (HIF) $\alpha$, which has many targets, e.g. VEGF, PDGF- $\beta$, erythropoietin, TGF- $\alpha$. VHL influences other cell functions (cell cycle, epithelial differentiation, extracellular fibronectin matrix) as well. More than 150 VHL germline mutations have been identified, and genotype-phenotype correlations led to the recognition of distinct VHL disease subtypes $(1,2 \mathrm{~A}, 2 \mathrm{~B}, 2 \mathrm{C})$. The major consequence of missing $\mathrm{pVHL}$ function is the continuous activation of HIF $\alpha$.

MET is a proto-oncogene, encoding a receptor tyrosine kinase that is normally activated by hepatocyte growth factor (HGF). The MET-HGF pathway is responsible for many critical regulatory cell functions. Most of the germline mutations occur in the MET activation loop or in the ATP-binding pocket, leading to ligand-independent MET activation.

FH encodes the mitochondrial Krebs cycle enzyme fumarate hydratase. About $40 \mathrm{FH}$ mutations have been described throughout the entire gene, without genotypephenotype correlation.

Birt-Hogg-Dubé (BHD) syndrome is a genodermatosis, and BHD gene encodes folliculin, without known function. Almost half of the mutations occur in the mononucleotide tract of cytosines due to slippage in the DNA polymerase during replication.
Genetic changes during tumor growth and progression

VHL gene, a key player in hypoxia-signaling pathway, is mutated or hypermethylated in $40-70 \%$ of sporadic clear cell RCC. It can be considered as a gate-keeper gene for RCC. In a high percentage of tumors from patients with sporadic clear cell RCC, one inherited allele of the VHL gene is mutant, and the second allele is deleted. In the presence of oxygen, $\mathrm{pVHL}$ forms a complex with other proteins, including elongin $\mathrm{C} / \mathrm{B}$, cullin 2, RBX1 (ROC/HRT1) and NEDD8, and as an ubiquitin ligase (E3) complex (VEC) targets the $\alpha$ subunit of HIF for ubiquitin mediated degradation. Adequate oxygenation causes proline hydroxylation of the HIF $\alpha$ subunit, and VHL protein binds to hydroxylated HIF $\alpha$ and directs attachment of a polyubiquitin chain. VEC also inhibit the binding of p300/CBP to HIF . When pVHL function is lost, or in hypoxia, HIF $\alpha$ is accumulated and transactivates many genes. Due to the overproduction of VEGF, the tumors associated with VHL disease are hypervascularized. Besides HIF $\alpha$, pVHL can ubiquitinate other proteins: protein kinase $\mathrm{C}$, VHL-interacting deubiquitinating enzymes (VDU), RNA polymerase-II-7 subunits (RPB7, RPB1). pVHL decreases the expression of matrix metalloproteases (e.g. MMP1), and increases the expression of their inhibitors (TIMPs). Without functioning pVHL, expression of p27 increased, while expression of cyclin D1 decreased in RCC cells. ${ }^{48}$

Somatic mutations of MET occur in $13 \%$ of sporadic papillary type-1 RCC. BHD somatic mutations are rare in sporadic RCC, but hypermethylation were observed in $\sim 30 \%$ of all RCC histological types. 
Staller et al. ${ }^{47}$ demonstrated that the chemokine receptor CXCR4 is a potential target of pVHL, and its high expression was associated with poor survival.

Carbonic anhydrase IX (CAIX) plays a role in the regulation of intra- and extracellular $\mathrm{pH}$ during periods of hypoxia in tumor cells. CAIX is one of the genes under the control of HIF-1 and seems to be very important in RCC. Tissue microarray (TMA) demonstrated that low CAIX staining was an independent predictor of poor survival of patients with metastatic RCC. Overall expression of CAIX decreased with development of metastases, according to the lower CAIX staining levels in metastatic lesions relative to matched primary tumor specimens. Furthermore, all the responders to IL-2 (8\%) included patients with high CAIX staining $(>85 \%)$ of the primary tumor. ${ }^{5,6}$ Another TMA study on clear cell RCC from 318 patients suggested, upon a multivariate analysis, that only metastatic status $(\mathrm{M}=1$, or $\mathrm{n}>0$ ), gelsolin, p53 and metastatic status/CAIX remained significant predictors of survival. ${ }^{19}$ In a similar analysis CAIX, vimentin and p53 were statistically significant predictors of survival independent of the clinical variables. ${ }^{22}$

A model using comparative genomic hybridization (CGH), a molecular screening method permitting the detection of all DNA losses and gains in a tumor, suggested that there may be at least two subclasses of clear cell RCC: one marked by the events $-6 \mathrm{q},+17 \mathrm{q},+17 \mathrm{p}$, and the other by $-9 p,-13 q,-18 q$. In papillary RCC the gains of $7 p$ and $17 \mathrm{p}$ are significantly higher in type 1 than in type 2 tumors. ${ }^{31}$ The transition of papillary adenoma to papillary $\mathrm{RCC}$ is accompanied by additional genetic alterations, ${ }^{20}$ however, it is not possible to make a distinction between adenoma and carcinoma purely by genetic changes, because many carcinomas show only few genetic alterations. It is a great challenge to find chromosomal alterations relevant to tumor progression. When copy number gains and losses were analyzed in RCC, only losses were significantly associated with a less favorable prognosis, presumably due to the inactivation of tumor suppressor genes. The loss of chromosome 9p suggested the involvement of p16INK4, but a mapping analysis strongly supported the existence of a hitherto unknown gene at $9 \mathrm{p} 13$. Other chromosomal changes with potential impact as predictors of outcome for RCC include losses of chromosome $8 \mathrm{p}$ and gains of $5 \mathrm{q} \cdot{ }^{31}$

\section{Studies on genetic or protein profiles}

Gene expression profiling is a promising tool to identify clinically relevant subclasses and to find novel markers of outcomes as survival and drug response. The integration of molecular markers defined by expression and proteomic profiling into the recently available prognostic systems (TNM staging, grade, functional status, etc.) is likely to further increase prediction accuracy.
Comparative genomic microarray analysis (CGMA) identified consistent downregulation of gene expression on chromosome $3 p$ and frequent upregulation on chromosome $5 \mathrm{q}$ in clear cell RCC samples, as well as gains in expression at chromosome $7,16 \mathrm{p}$ and 17 in papillary RCC. $^{50}$

Microarray technology revealed that RCC has a unique transcriptome. The glycogen content, known for long time for pathologists, is due to the overexpression of glycolytic enzymes such as enolase, phosphofructokinase and phosphoglycerokinase. Other, long known genetic features are the overexpression of EGFR/c-erbB1, VEGF and SOD2. On the other hand, genes that are under-represented include p57/kip2 suppressor gene, methallothionein and syndecan-1 proteoglycan. Interestingly, some genes involved in epithelial-mesenchymal transition (i.e. MUC18 and VCAM-1 adhesion molecules, LOX-1, laminin and collagen IV) are also overexpressed, suggesting that this feature may be involved in the progression of RCC.,27 The overexpression of several interferon-regulated genes (IIP-9-27, IFI-27, GBP2, MIG, IFI-16 and TAP-1) may explain the clinical benefit of IFN therapy in RCC.

It is interesting that gene expression profiling may not be limited to tumor tissue. Twine et al. ${ }^{51}$ claimed that expression profile of peripheral blood mononuclear cells can distinguish RCC patients from patients with other tumor types. The significance of this observation remains to be determined.

In a preliminary study on 29 samples of clear cell RCC, approximately 40 genes were suggested to discriminate between two groups of patients with different prognostic value based on 5 -year survival. ${ }^{49}$ The poor prognosis signature of RCC contained $\alpha 2$-macroglobulin, IGFBP7, TGFßRII, TIMP3 and phosphatidic acid phosphatase, while the good prognosis signature was defined by the expression of the oncogene FYN, oncosuppressor gene FAT, CDC42, autocrine motility factor, autotaxin (PDE1), the angiogenic cytokine PDGF- $\beta$ and vessel markers CD31 and CD34. This study also defined an immunodiagnostic set of proteins (vimentin, CD74, parvalbumin and galectin3 ) which can be used to differentiate between the classical $\mathrm{RCC}$, its chromophobic variant and the oncocytoma. In a larger study on 58 cases of stage IV RCC (unfortunately, of various subtypes grouped together), Vasselli et al. ${ }^{53}$ described a 45-gene signature for poor prognosis, highlighting VCAM-1 as a potential prognostic marker. Although these two studies differed in many respects, the lack of any overlap in the significant genes is striking. In general, the variability in expression profiling is a challenge for developing diagnostic and prognostic tools. Attempts for standardization of sample handling, microarray platform and statistical techniques are in progress.

The proteomic approach in RCC has only recently been introduced. Most of the studies used 2-D PAGE technique 
to compare the proteomes of malignant and benign samples. Some of the findings are as follows: $:^{25}$

- upregulated proteins in the serum of RCC patients: ferritin, erythropoietin, renin

- upregulated in tumor tissue: Mn-superoxide dismutase, cytokeratin 8 , major vault protein, thymidine phosphorylase, annexin I and IV, triosephosphate isomerase

- down-regulated in tumor tissue: enoyl-CoA hydratase, $\alpha$-glycerol-3-phosphate dehydrogenase, aldehyde dehydrogenase, aminoacylase I, stathmin, vimentin, lactate dehydrogenase $\mathrm{H}$-chain, agmatinase

The above list - the astonishing difference between the genomic and proteomic results - clearly indicates that we are just at the beginning to use these assays as useful tools in research or clinical aspects of cancer, specifically in RCC.

\section{Prognostic markers}

Classical negative prognostic factors of RCC are aneuploidy and high S-phase fraction. ${ }^{9}$ The classical clear cell RCC is characterized by p53 positivity in the majority of the cases $(70 \%)$, which was evaluated as negative prognostic factor, ${ }^{57}$ while telomerase does not have significance. ${ }^{29}$ Caveolin-1, considered to be an oncosuppressor, is frequently expressed by RCC but its increased presence serves as marker of less favorable outcome. ${ }^{7}$ BCL2 expression in RCC is frequent (70\%); it correlated with low Ki67 labeling index and rare p53 expression, and indicated a favorable prognosis. The expression of the apoptosis inhibitor XIAP is decreased with increasing grade of RCC, reflecting a poor prognosis. ${ }^{38}$

Two adhesion molecules, cadherin- 6 and MUC1/EMA ${ }^{36,45}$ have also been proved to be markers of poor prognosis in RCC, similarly to the protease ADAM $8 .{ }^{42}$ RCCs do not express estrogen or progesterone receptor, but in a small proportion of cases (15\%) androgen receptor can be detected, which tumors usually have poor prognosis. ${ }^{23}$ It is long known that RCC expresses EGFR, which is maintained in metastases. ${ }^{24}$ This is characteristic of the classical RCC, while the chromophobic variant is characterized by KIT expression. ${ }^{8}$ A marker of RCC, CAIX also serves as a marker of poor prognosis, ${ }^{5,6}$ similarly to spermine expression, although the molecular context is unrevealed. $^{41}$

Microarray analysis indicated the involvement of IGF/IGFR in RCC, and immunohistochemical studies further supported these observations, demonstrating IGFR in grade 3-4 cases. VEGF is constitutively present in the serum of RCC patients, but its level does not have prognostic significance, unlike the level of IL-6 indicating poor prognosis. ${ }^{33}$ On the other hand, VEGF can also be detected in RCC cells, and the intensity of staining correlates with the grade and stage. ${ }^{15}$

\section{Angiogenesis}

The classical RCC is characterized by the genetic/epigenetic inactivation of VHL, resulting in overexpression and enhanced function of HIF-1 $\alpha$. Due to the transcriptional activity of HIF-1 $\alpha$, growth factors such as VEGF and IGF, GLUT1 transporter, enzymes of glycolysis as well as CAIX are overexpressed. ${ }^{35}$ The upregulation of the HIF$1 \alpha$-VEGF axis results in the high level of angiogenic activity, ${ }^{56}$ producing one of the most vascularized cancer types in human, ${ }^{28}$ the level of which serves as marker of poor prognosis. ${ }^{18}$ Caveolin expression, shown to be a marker of unfavorable prognosis, seems to be associated with a higher vascular density as well. ${ }^{17}$

\section{Targeting genes and pathways}

There are several potential approaches for targeting the VHL pathway in VHL -/- RCC. One is to block HIF transcription e.g. by a camptothecin analog topotecan. Since it seems that HIF-2 is more critical to carcinogenesis than HIF-1, efforts are made to identify agents that can inhibit HIF-2. Another possibility is to target pathways downstream of HIF, e.g. VEGF, EGF, PDGF. Combination of agents that can inhibit at least two arms of the downstream HIF pathway, VEGFR and EGFR, are in clinical trials.

Angiosuppressive therapy is a form of targeted therapy. Since vascularization of cancer can be achieved by several alternative pathways (neoangiogenesis, vessel cooption, postnatal angiogenesis, vascular mimicry), targeting this process must be specific for the type of vascularization involved in a given cancer. RCC, one of the most vascularized cancers, ${ }^{28}$ and its clear cell subtype in particular, is characterized by a high microvascular density produced by tumor-induced neoangiogenesis. The molecular mechanism behind this strong angiogenic phenotype of clear cell $\mathrm{RCC}$ is the high frequency of the VHL inactivation leading to the upregulation of the HIF pathway and VEGF overexpression. ${ }^{40}$ It is important to note that VHL inactivation is a rare event in other common subtypes of RCC, accordingly, an angiosuppressive therapy may not be equally successful in those cases. Unfortunately in the published clinical trials of angiosuppressive therapies of RCC no such patient selection was made. ${ }^{40}$ Studies also indicated that VEGF expression and serum levels in clear cell RCC with or without VHL mutation is significantly different, suggesting another potential factor for patient selection. Meanwhile, the anti-VEGF antibody therapy of clear cell RCC on unselected patient population did not result in high frequency of objective response rates but expanded the time to progression by the frequent induction of stable disease. ${ }^{40}$ This observation may suggest that such an angiosuppressive regime serves better for metastasis prevention than an antitumoral one. Secondly, VEGF 
expression levels in RCC did not correlate with clinical responses ${ }^{40}$ further supporting the importance of the determination of the genetic characteristics of RCC responding to anti-VEGF therapy (VHL, p53, HIF status).

More recently, inhibitors of the VEGFR2 tyrosine kinase (TK), sutent (SU11248) and sorafenib (Bay-439006) demonstrated significant clinical activity against RCC, which was more pronounced compared to antiVEGF therapy. ${ }^{14,32}$ It is of note in this respect that, beside endothelial cells, RCC cells also express VEGFR2, providing an autocrine loop for proliferation control. ${ }^{40}$ Meanwhile, it is not known whether the responsiveness to VEGFR2-TK inhibitors depends on VEGFR2 expression or activity (phosphorylation) in RCC.

Activating point mutations in MET found in type 1 papillary RCC suggest at least three therapeutic strategies: blocking kinase activation with small molecule inhibitors of ATP binding, blocking HGF-MET interaction, or interactions between activated MET and downstream signaling molecules.

HSP90 in tumor cells forms a basis of a multichaperone complex that stabilizes and promotes the activity of a limited number of client proteins. HSP90 client proteins increased in RCC include HIF-1 $\alpha$ and receptor tyrosine kinases MET and KIT. KIT is overexpressed in chromophobe and papillary RCC, and its downstream targets as AKT and RAF are also HSP90 clients. HSP90 inhibitors, as 17-allylamino-17-desmethoxygeldanamycin, could be tools to disrupt the function of this multichaperone complex, resulting in a rapid inactivation of the client proteins.

The gold standard of therapy of progressing/metastatic RCC is immunotherapy using IL- 2 or IFN2 $(\alpha / \beta)$, even in the era of novel targeted therapies. However, little is known about markers that could predict the responsiveness of RCC to cytokine therapy. ${ }^{3,34}$ Analysis of the circulating and tumor-infiltrating leukocytes in RCC revealed that in responder cases the relative proportion of $\mathrm{T}$ cells increased (where the significance of $\mathrm{CD}^{+}$and $\mathrm{CD}^{+}$cells is still controversial $^{4}$ ), while those of the macrophages decreased in parallel with the decrease of circulating neutrophil leukocytes. ${ }^{12}$ On the other hand, the resistance of RCC to cytokine therapy was accompanied by increased circulating monocyte and neutrophil leukocyte levels. ${ }^{12,43}$ RCC sensitivity to IFN treatment was found to be associated with the induction of HLA I and -II expression, ${ }^{1}$ and may be mediated by $\mathrm{p} 53,{ }^{55}$ suggesting that the two forms of classical RCC ( $\mathrm{p} 53+/-)$ may not be equally appropriate for biotherapy. A further development in the field may come from the data obtained from global genomic studies revealing gene signature of clear cell RCC containing several IFN-responsive genes. ${ }^{2,27}$ The determination of the expression of IFN-responsive genes in the individual RCC, and analysis of the success of cytokine therapy may identify novel predictive markers for this type of therapy.
RCC is characterized by overexpression of $E G F R^{30,52}$ without mutation in the TK domain and rare amplification of the gene. ${ }^{44}$ However, immunohistochemical studies indicated high heterogeneity regarding intratumoral EGFR protein expression. ${ }^{24}$ Since both anti-EGFR antibody and EGFR-TK inhibitor therapies have been introduced in RCC management, ${ }^{11,13,16}$ it is mandatory to determine the diagnostic criteria for such treatments. The anti-EGFR antibody therapies require the expression of the wtEGFR with intact extracellular ligand-binding domain, while the EGFR-TK inhibitors require an active TK enzyme (autophosphorylation). Studies till now have not analyzed the clinical trials from this respect since the routinely used EGFR antibodies working on paraffin-embedded tissues recognize the near-transmembrane domain of EGFR at the extracellular side, therefore can only answer the question whether the EGFR protein is expressed by RCC, but are unable to provide further epitope information. Probably this is the reason why there was no correlation between EGFR protein expression in RCC and response to EGFRtargeted antibody- or TK inhibitor therapies. ${ }^{10}$

It is another issue how the histologic subtype of RCC affects the response to EGFR therapy. Classical clear cell RCC characterized by VHL inactivation has a lower level of EGFR expression than other subtypes, especially when compared to papillary RCC. In some trials subgroup analysis have been performed for papillary RCC and found that this type could be more sensitive for EGFR targeting than others. ${ }^{10}$

\section{Conclusion}

It is a strong hope that the more we characterize the pathways in an individual tumor, the better we will be able to evaluate the response to a specific therapy. Different array technologies could be powerful tools to achieve this goal, i.e. selecting patients on the basis of the genomic and/or proteomic profiles who would really benefit from the target-designed therapy. Genomic analysis of RCC accumulated ample of data which now can be exploited in the clinical management of a previously almost uncontrollable disease.

Genetic aberration of the VHL gene and its consequences on the function of HIF seem to play a fundamental role in RCC, resulting in a highly vascularized cancer which overexpresses, among other genes, VEGF. Probably this is the reason why anti-VEGF therapy proved to be successful against the advanced and therapy resistant disease. It is the hope that other genetic aberrations of RCC (MET, EGFR, HSP90) can also be exploited as drug targets. Even the discovery of IFN-responsive genes in the RCC-specific gene signature may call the attention to fine-tuning of the biotherapy of RCC (IFN or IL-2) in a more ,targeted” manner. 


\section{References}

1. Beniers AJ, Peelen WP, Debruyne FM, Schalken JA: HLAclass-I and -class-II expression on renal tumor xenografts and the relation to sensitivity for alpha-IFN, gamma-IFN and TNF. Int J Cancer 48: 709-716, 1991

2. Boer JM, Huber WK, Sültmann H, et al: Identification and classification on differentially expressed genes in renal cell carcinoma by expression profiling on a global human 31,500-element cDNA array. Genome Res 11:1861-1870, 2001

3. Bromwich E, McMillan DC, Lamb GW, et al: The systemic inflammatory response, performance status and survival in patients undergoing alpha-interferon treatment for advanced renal cancer. Br J Cancer 91:1236-1238, 2004

4. Bromwich EJ, McArdle PA, Canna K, et al: The relationship between T-lymphocyte infiltration, stage, tumour grade and survival in patients undergoing curative surgery for renal cell cancer. Br J Cancer 89:1906-1908, 2003

5. Bui $M H$, Seligson D, Han KR, et al: Carbonic anhydrase IX is an independent predictor of survival in advanced renal clear cell carcinoma: implications for prognosis and therapy. Clin Cancer Res 9: 802-811, 2003

6. Bui MH, Visapaa H, Seligson D, et al: Prognostic value of carbonic anhydrase IX and KI67 as predictors of survival for renal clear cell carcinoma. J Urol 171:2461-2466, 2004

7. Campbell L, Gumbleton M, Griffiths DF: Caveolin-1 overexpression predicts poor disease-free survival of patients with clinically confined renal cell carcinoma. Br J Cancer 89:1909-1913, 2003

8. Castillo M, Petit A, Mellado B, et al: C-kit expression in sarcomatoid renal cell carcinoma: potential therapy with imatinib. $\mathrm{J}$ Urol 171:2176-2180, 2004

9. Chautard D, Dalifard I, Chassevent A, et al: Prognostic value of UPA, PAI-1, and DNA content in adult renal cell carcinoma. Urol 63:1055-1060, 2004

10. Dancey JE: Epidermal growth factor receptor and epidermal growth factor receptor therapies in renal cell carcinoma: do we need a better mouse trap? J Clin Oncol 22: 2975-2977, 2004

11. Dawson NA, Guo C, Zak R, et al: A phase II trial of gefitinib (Iressa, ZD1839) in stage IV and recurrent renal cell carcinoma. Clin Cancer Res 10: 7812-7819, 2004

12. Donskov F, Bennedsgarrd KM, Hokland M, et al: Leukocyte orchestration in blood and tumour tissue following interlukin-2 based immunotherapy in metastatic renal cell carcinoma. Cancer Immunol Immunother 53: 729-739, 2004

13. Foon KA, Yand XD, Wiener LM, et al: Preclinical and clinical evaluations of ABX-EGF, a fully human anti-epidermal growth factor receptor antibody. Int J Radiat Oncol Biol Phys 58: 984-990, 2004

14. Gollob JA: Sorafenib: scientific rationales for single-agent and combination therapy in clear-cell renal cell carcinoma. Clin Genitourin Cancer 4: 167-174, 2005

15. Jacobsen J, Grankvist K, Rasmuson T, et al: Expression of vascular endothelial growth factor protein in human renal cell carcinoma. BJU Int 93:297-302, 2004

16. Jermann $M$, Stahel RA, Salzberg $M$, et al: A phase II, openlabel study of gefitinib (IRESSA) in patients with locally advanced, metastatic, or relapsed renal-cell carcinoma. Cancer Chemother Pharmacol 57: 533-539, 2006

17. Joo HJ, Oh DK, Kim YS, et al: Increased expression of caveolin-1 and microvessel density correlates with metastasis and poor prognosis in clear cell renal cell carcinoma. BJU Int 93:291-296, 2004

18. Kawata N, Yagasaki H, Hirakata H, et al: The impact of angiogenesis on the prognosis of advanced renal cell carcinoma. Hinyokika Kiyo 50:157-163, 2004
19. Kim HL, Seligson D, Liu X, et al: Using protein expression to predict survival in clear cell renal carcinoma. Clin Canc Res 10: 5464-5471, 2004

20. Kovacs $G$ : Molecular differential pathology of renal tumors. Histopathology 22: 1-8, 1993

21. Kovacs G, Akhtar M, Beckwith BJ, et al: The Heidelberg classification of renal cell tumors. J Pathol 183: 131-133, 1997

22. Lam JS, Belldegrun AS, Figlin RA: Tissue array-based predictions of pathobiology, prognosis, and response to treatment for renal cell carcinoma therapy. Clin Cancer Res 10: 6304s-6309s, 2004

23. Langner $C$, Ratschek $M$, Rehak $P$, et al: Steroid hormone receptor expression in renal cell carcinoma: an immunohistochemical analysis of 182 tumors. J Urol 171:611-614, 2004.

24. Langner $C$, Ratschek $M$, Rehak $P$, et al: Are heterogenous results of EGFR immunoreactivity in renal carcinoma related to non-standardised criteria for staining evaluation? J Clin Pathol 57:773-775, 2004

25. Lee $S$-W, Lee K-I, Kim JY: Revealing urologic diseases by proteomic technique. J Chromatogr B Analyt Technol Biomed Life Sci 815: 203-213, 2005

26. Linehan WM, Vasselli J, Srinivasan R, et al: Genetic basis of cancer of the kidney: disease-specific approaches to therapy. Clin Cancer Res 10, 6282s-6289s, 2004

27. Liou LS, Shi T, Duan Z-H, et al: Microarray gene expression profiling and analysis in renal cell carcinoma. BMC Urol 4:111,2004

28. Lörincz T, Tímár J, Szendrói M: Alterations of microvascular density in bone metastases of adenocarcinoma. Pathol Oncol Res 10: 149-153, 2004

29. Mekhail TM, Kawanishi-Tabata R, Tubbs R, et al: Renal cell carcinoma (RCC) and telomerase activity: relationship to stage. Urol Oncol 21:424-430, 2003

30. Moch H, Sauter G, Buchholz N, et al: Epidermal growth factor receptor expression is associated with rapid tumor cell proliferation in renal cell carcinoma. Hum Pathol 28: 1255-1259, 1997

31. Moch $H$ : Genomic alterations in renal tumours: what have we learned in the era of comparative genomic hybridisation? Pathology 36: 51-57, 2004

32. Motzer RJ, Michaelson D, Redman BG, et al: Activity of SU11248, a multitargeted inhibitor of vascular endothelial growth factor receptor and platelet-derived growth factor receptor, in patients with metastatic renal cell carcinoma. J Clin Oncol 24: 16-24, 2006

33. Negrier S, Perol D, Menetier-Caux $C$, et al: Interleukin-6, interleukin-10, and vascular endothelial growth factor in metastatic renal cell carcinoma: prognostic value of interleukin- 6 - from the Groupe Francais d'Immunothérapie. J Clin Oncol 22:2371-2378, 2004

34. Padrik $P$ : Prognostic factors of immunotherapy in metastatic renal cell carcinoma. Med Oncol 20:325-334, 2003

35. Pantuck AJ, Zeng G, Belldegrun AS, Figlin RA: Pathobiology, prognosis, and targeted therapy for renal cell carcinoma: exploiting the hypoxia-induced pathway. Clin Cancer Res 9:4641-4652, 2003

36. Paul R, Necknig U, Busch R, et al: Cadherin-6: a new prognostic marker for renal cell carcinoma. J Urol 171:97-101, 2004

37. Pavlovich CP, Schmidt LS: Searching for the hereditary causes of renal cell carcinoma. Nat Rev Cancer 4: 381-393, 2004

38. Ramp U, Krieg T, Caliskan E, et al: XIAP expression is an independent prognostic marker in clear-cell renal carcinomas. Hum Pathol 35:1022-1088, 2004

39. Richard S, Lidereau R, Giraud S: The growing family of hereditary renal cell carcinoma. Nephrol Dial Transplant 19: 29542958, 2004 
40. Rini BI, Small EJ: Biology and clinical development of vascular endothelial growth factor-targeted therapy in renal cell carcinoma. J Clin Oncol 23: 1028-1043, 2005

41. Rioux-Leclercq N, Delcros JG, Bansard JY, et al: Immunohistochemical analysis of tumor polyamines discriminates highrisk patients undergoing nephrectomy for renal cell carcinoma. Hum Pathol 35:1279-1284, 2004

42. Roemer A, Schwetmann L, Jung $M$, et al: Increased mRNA expression of ADAMs in renal cell carcinoma and their association with clinical outcome. Oncol Rep 11:529-536, 2004

43. Royston $P$, Sauerbrei $W$, Ritchie A: Is treatment with interferon- $\alpha$ effective in all patients with metastatic renal carcinoma? A new approach to the investigation of interactions. $\mathrm{Br} \mathrm{J}$ Cancer 90:794-799, 2004

44. Sakaeda T, Okumura N, Gotoh A, et al: EGFR mRNA is upregulated, but somatic mutations of the gene are hardly found in renal cell carcinoma in Japanese patients. Pharm Res 22: 17571761, 2005

45. Shimazui T, Yoshikawa K, Uemura H, et al: The level of cadherin- 6 mRNA in peripheral blood is associated with the site of metastasis and with the subsequent occurrence of metastases in renal cell carcinoma. Cancer 101:963-968, 2004

46. Staehler M, Rohrmann K, Haseke N, et al: Target agents for the treatment of advanced renal cell carcinoma. Curr Drug Targets 6: 835-846, 2005

47. Staller P, Sulitkova J, Lisztwan J, et al: Chemokine receptor CXCR4 downregulated by von Hippel-Lindau tumor suppressor pVHL. Nature 425: 307-311, 2003

48. Sufan RI, Jewett MAS, Ohh M: The role of von Hippel-Lindau tumor suppressor protein and hypoxia in renal clear cell carcinoma. Am J Physiol Renal Physiol 287: F1-F6, 2004
49. Takahashi M, Rhodes DR, Furge KA, et al: Gene expression profiling of clear cell renal cell carcinoma: gene identification and prognostic classification. Proc Natl Acad Sci USA 98: 9754-9759, 2001

50. Tan $M-H$, Rogers $C G$, Cooper JT, et al: Gene expression profiling of renal cell carcinoma. Clin Cancer Res 10: 6315s-6321s, 2004

51. Twine NC, Stover JA, Marshall B, et al: Disease-associated expression profiles in peripheral blood mononuclear cells from patients with advanced renal cell carcinoma. Cancer Res 63: 6069-6075, 2003

52. Uhlman DL, Nguyen P, Manivel JC, et al: Epidermal growth factor receptor and transforming growth factor alpha expression in papillary and nonpapillary renal cell carcinoma: correlation with metastatic behavior and prognosis. Clin Cancer Res 1: 913-920, 1995

53. Vasselli JR, Shih JH, Iyengar SR, et al: Predicting survival in patients with metastatic kidney cancer by gene-expression profiling in the primary tumor. Proc Natl Acad Sci USA 100: 6958-6963, 2003

54. Vogelzang NJ: Treatment options in metastatic renal carcinoma: an embarrassment of riches. J Clin Oncol 24: 1-3, 2006

55. Wittnebel S, Jalil A, Thiery J, et al: The sensitivity of renal cell carcinoma cells to interferon alpha correlates with p53-induction and involves Bax. Eur Cytokine Netw 16: 123-127, 2005

56. Yildiz E, Gokce G, Kilicarslan H, et al: Prognostic value of the expression of Ki-67, CD44 and vascular endothelial growth factor, and microvessel invasion, in renal cell carcinoma. BJU Int 93:1087-1093, 2004

57. Zigeuner $R$, Ratschek $M$, Rehak $P$, et al: Value of $\mathrm{p} 53$ as a prognostic marker in histologic subtypes of renal cell carcinoma: a systematic analysis of primary and metastatic tumor tissue. Urology 63:651-655, 2004 